$\checkmark$ Research Square

\title{
Leonurine Attenuates Pressure-Overload Cardiac Hypertrophy Induced By Abdominal Aortic Constriction In Rats
}

\section{Ding Xiaoli ( $\square$ awkk8@qq.com)}

Guizhou University Of Traditional Chinese Medicine https://orcid.org/0000-0002-3722-4898

\section{Yuan Qingqing}

Guizhou University Of Traditional Chinese Medicine

\section{Qian Haibing}

Guizhou University Of Traditional Chinese Medicine

\section{Research Article}

Keywords: Leonurine, cardiac hypertrophy, Rennin-angiotensin system, angiotensin II, angiotensin II type 1 receptor

Posted Date: May 27th, 2021

DOl: https://doi.org/10.21203/rs.3.rs-516132/v1

License: (c) (i) This work is licensed under a Creative Commons Attribution 4.0 International License. Read Full License 


\section{Abstract}

Background: Myocardial hypertrophy occurs in many cardiovascular diseases. Leonurine (Leo) is commonly used for cardiovascular and cerebrovascular diseases. However, whether it can prevent cardiac hypertrophy is not known. The aim of this study was to investigate the effect and mechanism of Leonurine (Leo) against pressure-overload cardiac hypertrophy induced by abdominal aortic constriction (AAC) in rats.

Methods: To answer this question, we prove it in the following way: Cardiac function was evaluated by hemodynamic; the left ventricle enlargement was measured by heart weight index (HWI) and left ventricular mass index (LVWI); myocardial tissue changes and myocardial cell diameter (MD) were determined by Hematoxylin and eosin (HE) staining; the $\beta$-myosin heavy chain( $\beta-\mathrm{MHC}$ )and atrial natriuretic factor (ANF), which are recognized as a marker of cardiac hypertrophy, were determined by Real-time quantitative PCR (qRT-PCR), then another gene phospholipase C (PLC), inositol triphosphate $\left(\mathrm{IP}_{3}\right)$, which associated with RAS were determined by Western blot(WB). angiotensin II (Ang II), angiotensin II type 1 receptor $\left(A T_{1} R\right)$ were determined by ELISA, WB and qRT-PCR methods. Finally, we measured the level of $\mathrm{Ca}^{2+}$ by microplate method and the protooncogene $\mathrm{c}$-fos and c-myc mRNA in left ventricular myocardium by qRT-PCR.

Results: Compare with control group, Leonurine can improve systolic dysfunction; inhibit the increase of left cardiac; inhibit myocardial cells were abnormally large and restrain the changes of cardiac histopathology; decrease the expression of $\beta-M H C, A N F, A n g ~ I I, A T_{1} R$, c-fos and c-myc mRNA and the protein levels of $\mathrm{PLC}, \mathrm{IP}_{3}$, Angll and $\mathrm{AT}_{1} \mathrm{R}$ in left ventricular myocardium, in addition, the content of $\mathrm{Ca}^{2+}$ also decrease.

Conclusion: Therefore, Leonurine can inhibit cardiac hypertrophy induced by AAC and its effects may be associated with RAS.

\section{Introduction}

Myocardial hypertrophy is one of the important causes of death of patients caused by cardiovascular diseases and an independent risk factor for the morbidity and mortality of cardiovascular diseases [1-3]. According to the investigation and research, the prevalence rate of hypertrophic cardiomyopathy in the Chinese population is $0.16 \%$, and the prevalence rate in males $(0.22 \%)$ is higher than that in females $(0.10 \%)$ [4]. Myocardial hypertrophy occurs in many cardiovascular diseases. Such as high blood pressure, coronary heart disease, valvular heart disease, congenital heart disease and cardiomyopathy, ect[5]. With time elapsing, the occurrence of cardiac hypertrophy can be divided into three stages: hypertrophy of the early, hypertrophy of the compensatory and the final decompensatory. Previous studies have shown that its development is closely related to cardiac overload[6], so abdominal aortic constriction(AAC) can use this principle to establish model of myocardial hypertrophy. If we allow this to continue, the results may lead to ventricular remodeling, decompensation of cardiac function and dilated 
cardiomyopathy, heart failure and even sudden cardiac death[7] Therefore, preventing the occurrence and development of cardiac hypertrophy is very important.

The rennin-angiotensin system (RAS) has been recognized to be an important factor in myocardial hypertrophy[8]. As a critical function of the system, Angiotensin II (Ang II) plays a key role in heart disease[9]. Ang II acts on angiotensin $\otimes$ type 1 receptor $\left(A T_{1} R\right)[10,11]$. $A T_{1} R$ is a member of the 7-fold transmembrane G-protein-coupled receptor (GPCR) family[12,13], mainly exists in the myocardial membrane and can mediate cardiac hypertrophy induced by pressure overload and mechanical stimulation after activation [14]. Ang II binds to $A T_{1} R$, which can induce vasoconstriction, increase blood pressure, and further induce cardiac hypertrophy and proliferation of fibroblasts [15]. and it may be related to the activation of PLC-IP3, PKC, JAK-STAT, MAPK, Ca/CaMK/CaN signaling pathways after Ang II binds to $A T_{1} R$ on the myocardium membrane. Here, we hypothesized that inhibit RAS may improve ACC rats anti hypertrophy caused by stress overload.

Leonurine (Leo) is one of the main active components of Chinese traditional medicine (Leonurus heterophyllus Sweet.) It is commonly used for cardiovascular and cerebrovascular diseases because it possesses anti-inflammatory analgesia, thrombolysis, improving microcirculation, antioxidant free radicals, reducing intracellular calcium overload, etc.previous studies have shown that leonurine had a certain therapeutic effect on the heart in acute myocardial infarction, myocardial ischemia and myocardial fibrosis in rats [16-20]. However, whether it can prevent or treat cardiac hypertrophy is not known. So, in this study, we investigated the potential therapeutic effects of Leonurine on Myocardial hypertrophy in stress overload using ACC rats and further its underlying mechanisms.

\section{Methods}

\subsection{Reagents}

Leonurine from Fudan University (Courtesy of Zhu Yizhen's Research Group), Losartan Potassium Tablet (Sichuan Hairong Pharmaceutical Co. Ltd), RNA preservation solution (Beijing Solaibao Biotechnology Co., Ltd), HE staining kit( Beijing Solaibao Biotechnology Co., Ltd), $\mathrm{Ca}^{2+}$ Content Kit( Nanjing Jiancheng Institute of Biological Engineering), Angiotensin $\otimes$ kit (nanjing institute of biological engineering), Angiotensin $\otimes$ receptor 1 kit (wuhan eli rhett biotechnology co., LTD), BCA Protein Quantitative Kit (Beijing Kangwei Century Biotechnology Co., Ltd), Animal total RNA rapid extraction kit (Chengdu Fuji Biotechnology Co., Ltd), RT EasyTM II (Chengdu Fuji Biotechnology Co., Ltd), EasyTM (Chengdu Fuji Biotechnology Co., Ltd), ANF Primer (Shanghai Sangon Biotechnology Technology Service Co., Ltd), $\beta$ MHC primer (Shanghai Sangon Biotechnology Technology Service Co., Ltd), Maker (Thermo), RIPA Lysis Buffer (Beijing Kangwei Century Biological Technology Co., Ltd), Protease Inhibitor (Beijing Kangwei Century Biotechnology Co. Ltd), SDS-Page Loading Buffer (Beijing Kangwei Century Biotechnology Co., Ltd), SDS-PAGE Gel Preparation Kit (Beijing Kangwei Century Biotechnology Co., Ltd), ECL High Sensitivity Chemiluminescence Detection Kit (Beijing Kangwei Century Biotechnology Co., Ltd), ECL Low Background Luminescence Detection Kit (Beijing Kangwei Century Biotechnology Co., Ltd), One-step 
Rapid WB(HRP) Kit (Rabbit) (Beijing Kangwei Century Biotechnology Co., Ltd), One-step Rapid WB(HRP) Kit (Rat) (Beijing Kangwei Century Biotechnology Co., Ltd), IP3 (abcam), PLC (abcam), AT1R (abcam), Angll (NOVUS), $\beta$-actin (Jingtiancheng Biotechnology (Beijing) Co., Ltd), RNA preservation solution (Beijing Solaibao Biotechnology Co., Ltd), Animal Total RNA Lsolation Kit (Foregene Inc), RT EasyTM II( Foregene Inc), Real Time PCR EasyTM (Foregene Inc).

\subsection{Animals and animal models}

Male Sprague-Dawley (SD) rats (200-220g body weight) were provided by Changsha Tianqin Biotechnology Co., Ltd., animal license number: SCXK (Hunan) 2014-0011. All animal experiments were performed in accordance with the Animal Care and Use Committee of Guizhou University of Traditional Medicine.

Abdonminal aortic banding (AAC), which is the rat model of cardiac hypertrophy, was produced through the method reported by Anversa [21]. Anesthesia with $1 \%$ sodium pentobarbital $(40 \mathrm{mg} / \mathrm{kg})$ intraperitoneal injection after animals fasting within $12 \mathrm{~h}$, but water is allowed. iodine volts routine disinfection of abdominal skin then opens abdominal cavity to separate abdominal aorta carefully. 4-0 surgical suture through just below the abdominal aorta and hit slipknot standby, a 7-gauge needle with blunt tip was placed in parallel abdominal aorta, which was ligated with the abdominal aorta by the 4-0 surgical suture. In the sham group, this same procedure was performed but no abdominal aorta was ligated. Then the viscera was restored to its original position and the surgical incision was closed. All animals in the sham operation group and model group were given intramuscular injection of penicillin sodium $(80,000 \mathrm{U}$ $/ 100 \mathrm{~g}$ ) for 3 consecutive days after operation. All rats were randomly divided into five groups: Sham group and Model group were given normal saline, the other groups were given corresponding doses of leonurine (30 $\mathrm{mg} \cdot \mathrm{kg}^{-1}$ and $15 \mathrm{mg} \cdot \mathrm{kg}^{-1}$ ) and positive drug (losartan potassium tablet, $5 \mathrm{mg} \cdot \mathrm{kg}^{-1}$ ) by intragastric administration, once a day, a total of 28 days.

\subsection{Hemodynamic detection method}

The BL-420Ss was used to record and collect data. After dieting forbidden and water for free for $12 \mathrm{~h}$, rats were anesthetized and fixed on the operating table. The right common carotid artery of rats was separated and the distal end of the common carotid artery was ligated with 4-0 surgical thread, the proximal end was clamped with artery clips. Then, insert the venous indwelling needle into the left ventricle. Record the left ventricular systolic pressure (LVSP), left ventricular end diastolic pressure (LVEDP), the rate of shrink/diastolic of left ventricular pressure $( \pm) \mathrm{dp} / \mathrm{dt}$ max $)$ after successful intubation for $15 \mathrm{~min}$.

\subsection{Determination of left ventricular hypertrophy index}

After measuring hemodynamic parameters, blot the excess water on the surface of the heart with a filter paper and weight it then separates the Left ventricle (including ventricular septal) and calculate the (Heart 
weight index, $\mathrm{HWI}$ ) and Left ventricular mass index (Left ventricular weight index, LVWI). According to the formulas:

$H W I=$ whole heart weight(HW)/ body weight(BW),

LVMI = left ventricle weight(LVW)/ body weight(BW).

\subsection{Hematoxylin and eosin (HE) staining}

The left ventricular tissue was immobilized in formalin, then dehydrated with different concentration alcohol, embedded in paraffin, sectioned with a thickness of $5 \mu \mathrm{m}$, dyed with HE. observed under the microscope and then calculated the Myocardial cell diameter.

\subsection{Determination of $\mathrm{Ca}^{2+}$, Ang冈and $\mathrm{AT}_{1} \mathrm{R}$ content}

The left ventricular myocardial tissue was added to PBS(1:9 weight to volume ratio), grind on ice. Then, the homogenate was centrifuged for 8 minutes, finally drawing the supernatant to test.

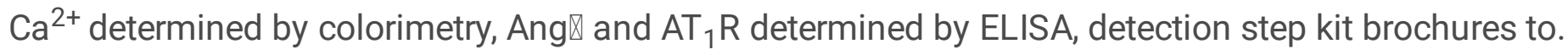

\subsection{Real-time PCR analysis}

Total RNA was extracted with Trizol. The concentration and purity of the extracted total RNA were determined by a micro nucleic acid analyzer. The cDNA was reverse-transcribed by PrimeScriptTM RT Reagent. Then the cDNA was amplified by Real time PCR using a real-time fluorescence quantitative PCR instrument. and the relative quantification was performed with internal reference as the standard. The mRNA relative expression levels of ANF, $\beta-M H C, A n g \rrbracket, A T 1 R, c-f o s, c-m y c$ in rat cardiac tissue were calculated by the $2^{-\triangle \triangle C T}$ method. The PCR primer sequences for rat ANF, $\beta-M H C, A n g \unrhd, A T{ }_{1} R, c$-fos, c-myc and housekeeping gene $\beta$-actin were designed and synthesized by Shanghai Sangon Biotechnology Technology Service Co., Ltd. The primer sequences were shown in Table 1.

Table. 1 Primer sequences for real-time quantitative RT-PCR 


\begin{tabular}{|lll|}
\hline Gene & Forward primer $\left(5^{\prime}\right.$-3' $\left.^{\prime}\right)$ & Reverse primer $\left(5^{\prime}\right.$-3') \\
\hline ANF & CCAGAGAGTGAGCCGAGACAGCAAAC & CTCCTCCAGGTGGTCTAGCAGGTTCT \\
\hline B-MHC & GCGAGCTGGCTAGGCAACTGGAAGA & TGGCGTCCGTCTCATACTTGGTCCTC \\
\hline c-fos & GTGGACCTGTCTGGTTCCTTCTATGC & CTCGTTGCTGCTGCTGCCCTTT \\
\hline c-myc & CACCGCCTACATCCTGTCCGTTCAA & CGAGATTCCAGCTCCTCCTCACTTCC \\
\hline Angll & GGCAAATCTGGGCAAGATGGGTGACA & TGGCGAACAGGAACGGACTGCTCAG \\
\hline AT ${ }_{1}$ R & CCAAGGCTGGCAGGCACAGTTACAT & ACACTGGCGTAGAGGTTGAAGCTCAC \\
\hline$\beta$-actin & GAAGATCAAGATCATTGCTCCT & TACTCCTGCTTGCTGATCCA \\
\hline
\end{tabular}

\subsection{Western blot analysis}

The myocardial tissue of rats homogenizer homogenate cracking in low temperature, protein extraction kit was used to extract total protein, sample concentration with protein content detection kit determination method (BCA), with SDS denaturant, boil degeneration, after SDS-PAGE, using constant pressure turn wet method, $120 \mathrm{v}$, transfer film $2 \mathrm{~h}$, the protein on the gel strips to PVDF membrane. The PVDF membrane was closed by slow shaking in 3\%BSA solution at room temperature for $2 \mathrm{~h}$, and then incubated overnight with $A n g \rrbracket, A T_{1} R, P L C, I P_{3}$ total protein antibodies at $4^{\circ} \mathrm{C}$, respectively. After washing with TBST 5 times, then incubate the secondary antibody for $1.5 \mathrm{~h}$. After washing with TBST 5 times, use the ECL to chemiluminescent and use Imaging System to image.

\section{Results}

\subsection{Effects of leonurine on hemodynamics in AAC-induced rats with ventricular hypertrophy}

Hemodynamic examination was used to determine the parameters of cardiovascular function. Compared with sham group, LVSP and LVEDP were significantly increased $(p<0.01)$ in model group, $(+) \mathrm{dp} / \mathrm{dt}_{\text {max }}$ and $(-) \mathrm{dp} / \mathrm{dt}_{\max }$ were decreased $(p<0.01)$, Leonurine treatment, however, dramatically reduced the level of LVSP and LVEDP $(p<0.05)$, improved $(+) \mathrm{dp} / \mathrm{dt}_{\max }$ and $(-) \mathrm{dp} / \mathrm{dt}_{\max }(p<0.05)$. These results showed that Leo30 and Leo-15 groups can improve the systolic dysfunction in rats with cardiac hypertrophy induced by AAC.(Table 2)

Table. 2 Effects of leonurine on hemodynamics in AAC-induced rats with ventricular hypertrophy 


\begin{tabular}{|llllll|}
\hline Group & Dose & LVSP & LVEDP & $(+) \mathrm{dp} / \mathrm{dtmax}$ & $(-) \mathrm{dp} / \mathrm{dtmax}$ \\
& $/ \mathrm{mg}^{\mathrm{kg}}{ }^{-1}$ & /mmHg & /mmHg & $\mathrm{mmHg} \cdot \mathrm{s}^{-1}$ & $\mathrm{mmHg} \cdot \mathrm{s}^{-1}$ \\
\hline Sham & - & $172.5 \pm 34.53$ & $10.00 \pm 1.32$ & $6693.12 \pm 198.02$ & $6443.50 \pm 347.21$ \\
\hline Model & - & $190.15 \pm 9.89^{\# \#}$ & $32.24 \pm 2.41^{\# \#}$ & $4548.75 \pm 238.22^{\# \#}$ & $4305.87 \pm 84.88^{\# \#}$ \\
\hline Losartan & 5 & $179.09 \pm 6.27^{\star}$ & $24.50 \pm 3.26^{*}$ & $4784.37 \pm 174.77^{\star}$ & $4530.75 \pm 92.02^{*}$ \\
\hline Leo-30 & 30 & $171.42 \pm 7.30^{\star}$ & $16.15 \pm 1.34^{*}$ & $5720.12 \pm 261.32^{*}$ & $5466.25130 .52^{*}$ \\
\hline Leo-15 & 15 & $182.73 \pm 4.79^{\star}$ & $22.95 \pm 2.19^{\star}$ & $4802.00 \pm 111.80^{*}$ & $4572.75 \pm 79.83^{*}$ \\
\hline
\end{tabular}

LVSP,left ventricular systolic pressure, LVEDP,left ventricular end-diastolic pressure, $\pm d p / d t m a x$, maximum increase and decrease in left ventricular pressure.Data are means $\pm s . d, n=8$ rats. \#\#p[0.01 vs Sham group; ; $[0.05$ vs Model group.

\subsection{Effects of leonurine on AAC-induced left cardiac hypertrophy}

As is shown in Fig.1A-B. Left cardiac hypertrophy was a compensatory change to maintain normal pumping function of the heart in the early stage of heart failure. Compared with the sham group, the model group was found to be markedly higher in HWI and LVWI $(p<0.01)$, the Leo-30 and Leo-15 groups can inhibit left ventricular hypertrophy $(p<0.05)$. These results indicate leonurine can decrease the increase of left cardiac to protect the heart.

\subsection{Effects of leonurine on the tissue in left ventricular myocardium}

As shown in sham group, Cardiomyocytes exhibited the typical pattern of short cylindrical and cell nucleus located in the center orderly by HE staining. In contrast, HE-stained sections from the model group revealed myocardial cells were swelling and degeneration and significantly thicker of chromatin, increased of connective tissue and inflammatory cell infiltration. Alternatively, Leo-30 and Leo-15 groups reduced the inflammatory cell infiltration compared to the model group and partially restored the order of Cardiomyocytes (Fig.2)

\subsection{Effects of leonurine on cardiac myocyte diameter}

Previous studies confirmed that myocardial cells are the basic units of heart contraction and relaxation, cardiac dysfunction is often associated with an increased MD. In this search, we found that the MD of the model group was significantly increased $(p<0.01)$, indicating the hypertrophy of rat cardiomyocytes after AAC. Compared with the model group, MD of rats in Leo-30 and Leo-15 groups were significantly decreased $(p<0.05)$, suggesting that Leonurine may inhibit myocardial cells were abnormally large to protect the heart.(Fig.3)

\subsection{Effects of Leonurine on the contents of Angll, $\mathrm{AT}_{1} \mathrm{R}$ and $\mathrm{Ca}^{2+}$ in myocardial tissue}


Up-regulated expressions of Angll, $\mathrm{AT}_{1} \mathrm{R}$ and $\mathrm{Ca}^{2+}$ have been recognized as an important cause of cardiac hypertrophy. The results of colorimetry indicated that the level of Angll, $A T_{1} R$ and $\mathrm{Ca}^{2+}$ were significantly increased of model group in comparison with the sham group $(p<0.01)$. However, we also found that treatment with leonurine inhibited the increase of Angll, $\mathrm{AT}_{1} \mathrm{R}$ and $\mathrm{Ca}^{2+}$, especially in Leo-15 group $(p<0.05)$. These results suggest that leonurine can decrease the level of $A n g l l, \mathrm{AT}_{1} \mathrm{R}$ and $\mathrm{Ca}^{2+}$ to inhibit cardiac hypertrophy. (Fig.4A-C)

\subsection{Effects of leonurine on the expression of $\beta-M H C, A N F, c-f o s, c-m y c$, Angll and AT1R mRNA in left ventricular myocardium}

Using quantitative RT-PCR for detection of mRNA expression, our results show that the expression of $\beta$ MHC, ANF, c-fos, c-myc, Angll and AT1R mRNA significantly increased in the model group $(p<0.01), \beta-M H C$ and ANF are currently recognized as the signature genes of myocardial hypertrophy. Therefore, combined with these results, it was concluded that the model of myocardial hypertrophy induced by abdominal aortic constriction in rats was successfully replicated. Compared with model group, Leo-30 and Leo-15 groups decreased $\beta$-MHC, ANF, c-fos, c-myc, Angll and AT1R mRNA express $(p<0.05)$. The results suggest that leonurine can inhibit their expression level, thereby inhibiting the occurrence of AAC postoperative myocardial hypertrophy in rats. (Fig.5A-F)

\subsection{Effects of leonurine on the expression of Angll, $A T_{1} R, P L C$ and $\mathrm{IP}_{3}$ protein in left ventricular myocardium}

PLC-IP ${ }_{3}$ signaling pathway was significant in the heart. The increase of PLC activity can trigger a series of injury responses and promote the occurrence of myocardial hypertrophy. We measured the Angll, $A T_{1} R$, $\mathrm{PLC}, \mathrm{IP}_{3}$ expression level using Western blot. The data demonstrated that the Angll, $A T_{1} R, P L C, \mathrm{IP}_{3}$ level in the model group was significantly higher than that in the control group $(p<0.01)$. But after the treatment of Leonurine, the level of Angll, $A T_{1} R, P L C$ and IP $\mathrm{P}_{3}$ protein decreased $(p<0.05)$, so inhibiting these proteins may protect the heart. (Fig.6A-D)

\section{Discussion}

In recent years, the prevalence and mortality of heart failure caused by cardiac hypertrophy in cardiovascular diseases in China have been on the rise [21] and cardiac pressure overload is the main pathological basis of Myocardial hypertrophy. (Abdominal Aortic Coarctation, AAC) is a commonly used model for building cardiac hypertrophy caused by cardiac pressure overload [22]. This method is relatively simple to operate and reduces postoperative mortality in animals. So in our current study, we used this model to observe if leonurine can inhibit cardiac hypertrophy. The results demonstrated that leonurine can significantly inhibit myocardial hypertrophy of AAC-induced rats. These beneficial effects were partially associated with 1 . improve systolic dysfunction 2 . inhibit the increase of left cardiac 3 . inhibit myocardial cells were abnormally large and restrain the changes of cardiac histopathology 
4.decrease the expression of $\beta-M H C$, ANF mRNA which is recognized as a marker of cardiac hypertrophy. 5. modulate RAS and correlation factor

Elevated transcription of ANF, BNP, a-MHC and $\beta-M H C$ mRNA have been proved to be closely related to myocardial hypertrophy[23-26]. When heart disease occurs, the expression of a-MHC is down-regulated, while the expression of $\beta-\mathrm{MHC}$ is increased, thus transforming the relatively stable adult gene to the fetal gene with certain proliferation then accelerating the proliferation and differentiation of cardiomyocytes [27]. This search found that leonurine inhibits the expression of $\beta-M H C, M H C$, ANF mRNA induced by AAC, so which proved that leonurine can inhibit cardiac hypertrophy. From the hemodynamic indicators shows that compared with the control group, LVSP and LVEDP were decreased, $(+) \mathrm{dp} / \mathrm{dtmax}$ and (-)dp/dtmax were increased in all groups with leonurine. So these results imply leonurine by means of improving the systolic dysfunction to inhibit cardiac hypertrophy. Similarly, leonurine inhibit the rise of LWI, LVHI to inhibit cardiac hypertrophy.

Not any way. HE staining also shows that all the dose of leonurine groups could improve the pathological changes of myocardial cell swelling, degeneration, nuclear enlargement, chromatin thickening, intermittent widening of muscle bundle, disordered arrangement, connective tissue hyperplasia and inflammatory cell infiltration, and reduce the diameter of myocardial cell (MD). So these results imply leonurine can through this way to inhibit cardiac hypertrophy.

RAS is an important endocrine regulation mechanism of the body, which consists of renin, angiotensin and their receptors. It plays an extremely important role in the regulation of blood pressure and cardiovascular disease, and is the main pathological mechanism of cardiac hypertrophy [28]. The occurrence and development of cardiac hypertrophy is mainly closely related to local cardiac RAS [29-30]. Ang 『is the key to active RAS, the rise of Ang $₫$ was further caused the left ventricular pressure overload, so as to induce the body left ventricular hypertrophy [31]. Ang $\otimes$ of $A T_{1} R$ is highly selective[32]. We used ELISA to analyse the level of Angll and $A T_{1} R$ in myocardial tissue and found that Leonurine can decrease the level of Angll and $\mathrm{AT}_{1} \mathrm{R}$. Then we tested again by Western Blotting and quantitative RT-PCR. The results are the same as before. So these results further illustrate the leonurine may access modulate RAS to effect myocardial hypertrophy. Ang $\otimes$ and $A T_{1} R$ height on the myocardial cell membrane for a long time, can lead to myocardial cell hypertrophy and hyperplasia, inflammation and fibrosis.

Ang II also can increase $\mathrm{Ca}^{2+}$ concentration in cardiomyocytes and promote proliferation of cardiomyocyte when inducing cardiomyocyte hypertrophy model in vitro [33], or induce cardiomyocyte hypertrophy by increasing $\mathrm{Ca}^{2+}$ content in mitochondria of cardiomyocytes and promoting the expression of calmodulin-dependent protein kinase II in cardiomyocytes [34-35]. In this search, we found that leonurine decreased the level of $\mathrm{Ca}^{2+}$. These results imply leonurine inhibits cardiac hypertrophy also associated with $\mathrm{Ca}^{2+}$. It may mediate the occurrence and development of cardiac hypertrophy by regulating the content of $\mathrm{Ca}^{2+}$ and $\mathrm{Ca}^{2+}$-related signaling pathways in the myocardium. 
In addition, activation of RAS may activate PLC-IP ${ }_{3}$, DAG-PKC and JAK/STAT 3 signaling pathways[36]. Phospholipase $\mathrm{C}(\mathrm{PLC})$ is a major enzyme on $\mathrm{PLC}^{-\mathrm{IP}_{3}}$ signal pathway, which can catalytic hydrolysis phosphodiester linkage. Current studies show that the increase of PLC activity can promote the occurrence of cardiac hypertrophy [37]. This pathological process is mainly due to the activated PLC acting on phosphatidylinositol bisphosphate $\left(\mathrm{PIP}_{2}\right)$, producing inositol trisphosphate $\left(\mathrm{IP}_{3}\right)$ and diacylglycerol (DAG), then $\mathbb{I P}_{3}$ act on $\mathrm{IP}_{3}$ receptor $\left(\mathrm{IP}_{3} \mathrm{R}\right)$ in the endoplasmic reticulum promote the release of $\mathrm{Ca}^{2+}$ into the cytoplasm to lead the increase of intracellular $\mathrm{Ca}^{2+}$ concentration, which further triggers the transcriptional expression of proto-oncogenes c-myc and c-fos, increases the content of DNA and RNA in cardiomyocytes, increases protein synthesis, promotes the generation and differentiation of cardiomyocytes, and finally induces ventricular remodeling [39-40].

In recent years, many scholars have found that the abnormal expression of proto-oncogenes in the body is closely related to the occurrence and development of cardiovascular diseases, and it is believed that the abnormal expression of c-fos and c-myc genes may be the main pathogenic factors of cardiac hypertrophy, hypertension and myocardial ischemia [41]. Both c-fos and c-myc belong to a class of intraconuclear oncogenes, which have a high expression under cardiac stress overload [42]. Shen Juanjuan [43], Li Chunling [44] and other cells induced by Ang II myocardial hypertrophy model found in the process of proto-oncogenes c-fos, c-myc in myocardial cells have higher expressions in the model group, so as to reveal the original cancer gene the expression of c-fos and c- myc involved in the development of myocardial hypertrophy. In our research, compared with sham groups, the expression of $\mathrm{PLC}, \mathrm{IP}_{3}$ protein and c-fos, c-myc mRNA was increased in rats with cardiac hypertrophy induced by AAC. which proved the importance of PLC, IP 3 , c-fos, c-myc in cardiac hypertrophy. Even though leonurine can down-regulate the expression of PLC, IP 3 protein and c-fos, c-myc mRNA to inhibit cardiac hypertrophy and its effect may be associated PLC-IP 3 signaling pathways as well as c-fos, c-myc.

To sum up, leonurine can inhibit cardiac hypertrophy induced by AAC. Its effects may be associated with RAS. However, the pathogenesis of cardiac hypertrophy is diverse, including not only RAS but also the sympathetic nervous system, oxidative stress, mitochondrial damage, catecholamine, endothelin and other cytokines. The results of this study proved hypertrophy can regulate RAS to treat cardiac hypertrophy. Whether the improved cardiac hypertrophy observed with leonurine is due to other mechanisms remains elusive, and needs further investigation.

\section{Declarations}

Acknowledgements organized by Guizhou university of traditional Chinese medicine and Zhu Yichun research group

Author Contributions Ding xiaoli was responsible for writing the first draft and analysis. Yuan qingqing was responsible for designing the experiment. Qian haibing was responsible for finding the problem. 
Funding This work was supported by National Natural Science Foundation of China(grant number 8176077)

Data Availability Not applicable

\section{Compliance with Ethical Standards}

Conflict of interest The authers declare no competing interests

Consent to participate Not applicable

Consent to publication Not applicable

Code Avaiability Not applicable

\section{References}

1. Umar S, Nadadur R, lorga A, et al. Cardiac structural and hemodynamic changes associated with physiological heart hypertrophy of pregnancy are reversed postpartum[J]. J Appl Physiol, 2012, 113(8): 1253-1259.

2. Diwan A, Dorn GW 2nd. Decompensation of cardiac hypertrophy: cellular mechanisms and novel therapeutic targets[J]. Physiology, 2007, 22: 56-64.

3. Berenji K, Drazner MH, Rothermel BA, et al. Does load-induced ventricular hypertrophy progress to systolic heart failure[J]. Am J Physiol Heart Circ Physiol, 2005, 289(1): H8-H16.

4. A review of the effects of the treatment on the risk of heart disease [J]. J Clin Endocrinol, 2011, 35 (2): 207-216

5. Li P. Effects of rosuvastatin on PPARY and NF-KB protein expression in rats with cardiac hypertrophy [D].Liaoning Medical College, 2012.

6. Miyata S, Minobe W, Bristow M R, et al. Myosin heavy chain isoform expression in the failing and nonfailing human heart[J]. Circulation research, 2000, 86(4):386-390.

7. Maillet M, van Berlo JH, Molkentin JD. Molecular basis of physiological heart growth: fundamental concepts and new players[J]. Nat Rev Mol Cell Biol, 2013 14(1): 38-48.

8. Zhang B, Jiang J, Yue Z, Liu S, Ma Y, Yu N, Gao Y, Sun S, Chen S and Liu P. Store-Operated Ca2+ Entry (SOCE) contributes to angiotensin II-induced cardiac fibrosis in cardiac fibroblasts. JPharmacol Sci 2016; 132: 171-180.

9. Wang Y. Study on the mechanism of Ophiopogon saponin D inducing CYP2J production against myocardial hypertrophy [D].Guangxi Medical University, 2017

10. Li S, Huang B, Zhou C, Shi J, Wu Q, Jiang Q. Rutaecarpine Ameliorates Pressure Overload Cardiac Hypertrophy by Suppression of Calcineurin and Angiotensin II [J]. Evid Based Complement Alternat Med. 2021;1(12) 1-8. 
11. SHANMUGAM P, VALENTE A J, PRABHU S D, et al.Angiotensin- $\$ type 1 receptor and NOX2 mediate TCF/LEF and CREB dependent WISP1 induction and cardiomyocyte hypertrophy[J].JMol Cell Cardiol, 2011, 50(6):928-938.

12. Ming Y. SNF5 impact on angiotensin $\triangle$ induced myocardial hypertrophy of experimental research [D].Qingdao University, 2017.

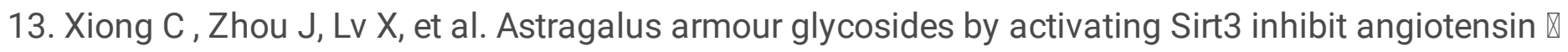
induced myocardial hypertrophy and oxidative stress [J].Central South Pharmacy, 2019, 17(01): 3437.

14. Gong H. Angiotensin $\otimes$ receptor activation with heart damage repair $[A]$.

15. Luo M. Study on the effect of sodium ferulate on myocardial hypertrophy in rats [D]. Zunyi Medical College, 2017.

16. National Pharmacopoeia Commission. The First Pharmacopoeia of the People's Republic of China [M].Beijing: China Medical Science and Technology Press, 2015, 290-291.

17. Loh KP囚Huang SH囚Tan BK囚Cerebral protection of purified Her-ba Leonurine extract on middle

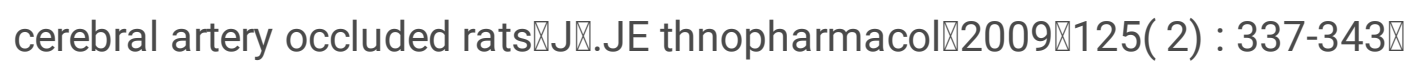

18. Xu Y, Qian H , Zhu Y . Effect of Leonurine alkaloid on acute myocardial infarction in rats [J].Chinese Journal of Experimental Formulae, 2016,22(17): 113-116.

19. Xu Y, Qian H. Experimental study on mothermotherine in rats with heart failure after infarction [J].Modern Distance Education of Traditional Chinese Medicine in China, 2015, 13(11): 144-145.

20. Li M. Study on the effect and mechanism of Leonuri alkaloid on myocardial fibrosis [D]. Guiyang College of Traditional Chinese Medicine, 2017.

21. Zhang $R$, Zhang $Y Y$, Huang $X R$, et al. C-reactive protein promotes cardiac fibrosis and infiammation in angiotensin II-induced hypertensive cardiac disease[J]. Hypertension, 2010, 55『4囚: 953-960.

22. Liao J, Xia Y. Effects of allicin on left ventricular hypertrophy and calcineurin signaling pathway in rats with abdominal aortic coconstriction [J].China Medical Review, 2012, 9(05): 18-20.

23. Tang CM, Liu FZ, Zhu JN, et al. Myocyte-specific enhancer factor 2C: a novel target gene of mi R-214$3 p$ in suppressing angiotensin II-induced cardiomyocyte hypertrophy[J]. Sci Rep, 2016, 6: 36146.

24. Yang $X$, Tian J, Chen $G$. Histone acetylase subtype $P 300$ regulates the dynamic expression of cardiac specific transcription factors during mouse embryonic heart development [J].Journal of Chongqing Medical University, 2010, 35(7): 961-965.

25. Jiang D, Liu L, Lu T, et al. CBP/P300 mediated acetylation of histone H3K9 and its effect on GATA4 expression [J].Journal of the Third Military Medical University, 2016, 38(18): 2018-2022.

26. Hang CT, Yang J, Han P, et al. Chromatin regulation by Brg1 underlies heart muscle development and disease [J]. Nature,2010, 466(7302): 62-67.

27. Mehrotra A, Joe B, De La Serna IL. SWI/SNF chromatinremodeling enzymes are associated with cardiac hypertrophyin a genetic rat model of hypertension [J]. J Cell Physiol,2013, 228(12): 23372342. 
28. Tian B, Gao G, Lu X. The role of local renin-angiotensin system in volumetric overload myocardial hypertrophy in rats [J].Chinese Journal of Pathophysiology, 1993(02): 116-120.

29. Patten RD, Aronovitz MJ, Einstein M, et al. Effects of angiotensin II receptor blockade versus angiotensin-converting-enzyme inhibition on ventricular remodelling following myocardial infarction in the mouse[J]. Clin Sci (Lond), 2003, 104(2): 109-118.

30. Wang W. Effects of ginsenoside RB-1 on renin-angiotensin system in rats with left ventricular remodeling after myocardial infarction [D]. Huazhong University of Science and Technology, 2006.

31. Su J, Li C, Su Z. From the Foundation to the Clinical Clinic [M].Beijing: Beijing Medical University and China Union Medical College Press, 1998. 372-381.

32. Sadoshima J,Izumo S.Signal transduction pathways of angiotensin囚-in-duced c-fosgene expression in cardiac myocytes in vitro.Rolesof phospho-lipid-derived second messengers[J]. Circ Res, 1993, 73(3): 424-438.

33. Jiang DS, Zhang XF, Gao L, et al. Signal \egulatory Protein-a Protects Against Cardiac Hypertrophy Via the Disruption of Toll -Like 囚eceptor 4Signaling[J]. Hypertension, 2014, 63( 1) : 96-104】

34. Yang, T. The mitochondrial calcium intake of protein in 1 angiotensin $\otimes$ induced myocardial hypertrophy of the pathological changes in mice protection [J].Medical Journal of the PLA, 2017, 42(12): 1051-1055.

35. Luo M, Zhang H, Zhao L. Calcium/calmodulin dependent protein kinase $₫$ cardiac mast cells in rat embryo in regulating cell autophagy induced by angiotensin $\mathbb{[}$ [J].Journal of Applied Medicine, 2017, 33(12): 1917-1922.

36. Sadoshima J,Izumo S.Signal transduction pathways of angiotensin囚-in-duced c-fosgene expression in cardiac myocytes in vitro.Rolesof phospho-lipid-derived second messengers[J]. Circ Res, 1993, 73(3): 424-438.

37. Sun $Y$, Wang $S$, Cai H. Changes in the activity of cardiolipinase $C$ in hypertrophy and the effect of captopril on it [J].Journal of Henan Medical Research, 2001(01): 17-19.

38. Zhang B, Tang J, Yang L, et al. Effects of rosuvastatin combined with irbesartan on myocardial hypertrophy remodeling in rats [J].Chinese Journal of Pathophysiology, 2016, 32(03): 534-538+543.

39. Li L. $\beta$-adrenergic receptor-activated protein kinase $C-\varepsilon$ induces cardiomyocyte hypertrophy and its mechanism [D].Kunming Medical University, 2011.

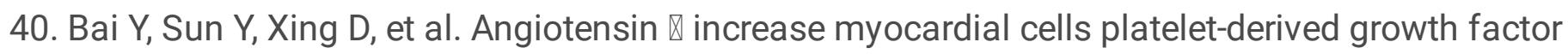
receptor - beta [J].Chinese Science Bulletin, 2001(17): 1450-1453.

41. Zhang M. Effects of Yixin Shu on left ventricular hypertrophy and c-fos and c-myc gene expression in renal hypertensive rats [D].Heilongjiang University of Chinese Medicine, 2005.

42. Tang Xi, Yan C.Study on the proto-oncogenes associated with left ventricular hypertrophy and the reversible effect of traditional Chinese medicine [J].Science and Technology of Chinese Medicine, 2000(03): 180-181. 


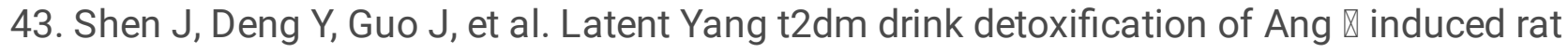
cardiomyocyte hypertrophy proto-oncogene $\mathrm{c}$ fos, c myc mRNA influence [J].Chinese Journal of Traditional Chinese Medicine, 2014, 29(11): 3543-3546.

44. Li C, Gu G, Luo J, et al. Effects of Yiwengyang Huoxue Prescribe on the expression of c-fos and cmyc in rat hypertrophic cardiomyocytes [J].Chinese Journal of Gerontology,2014,34(17):4897-4899.

Figures
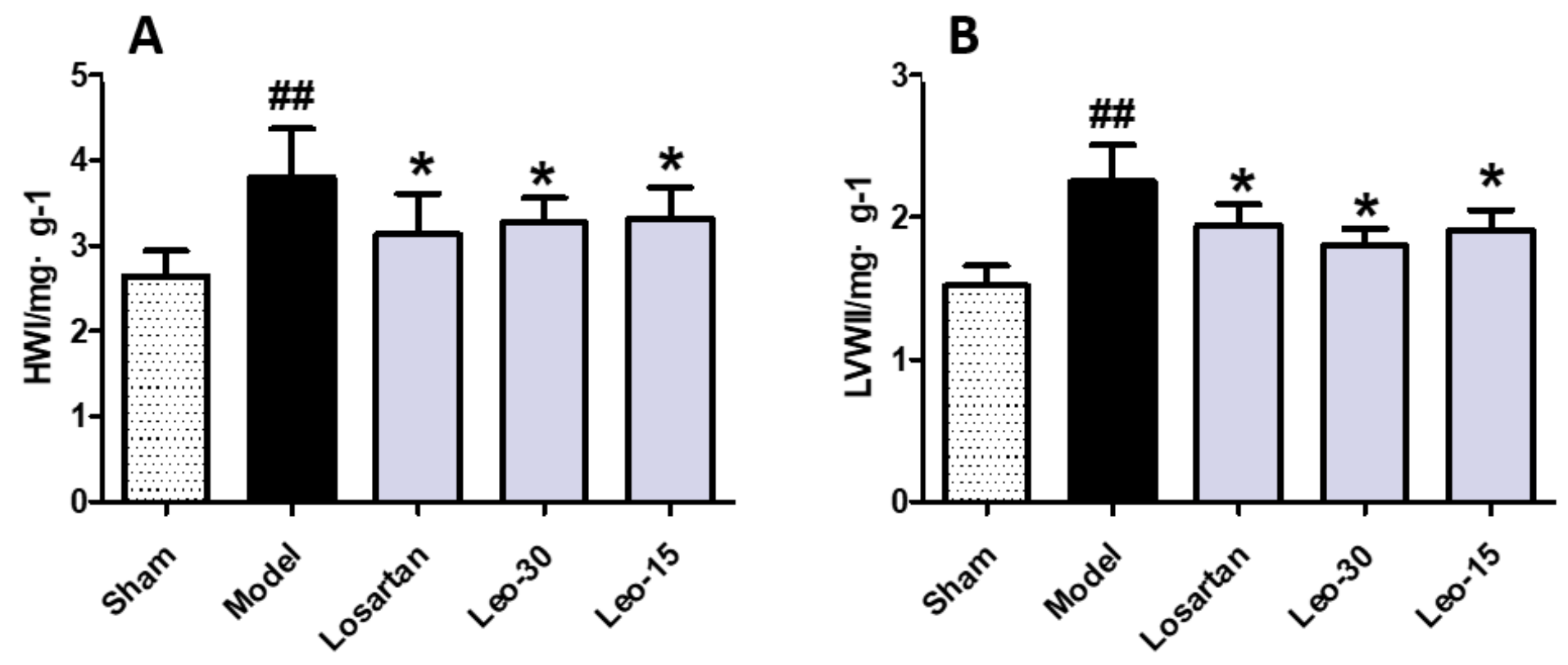

Figure 1

Effects of leonurine on AAC-induced left cardiac hypertrophy HWI, heart weight index, LVWI, left

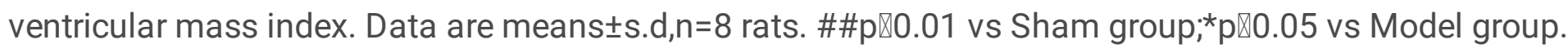



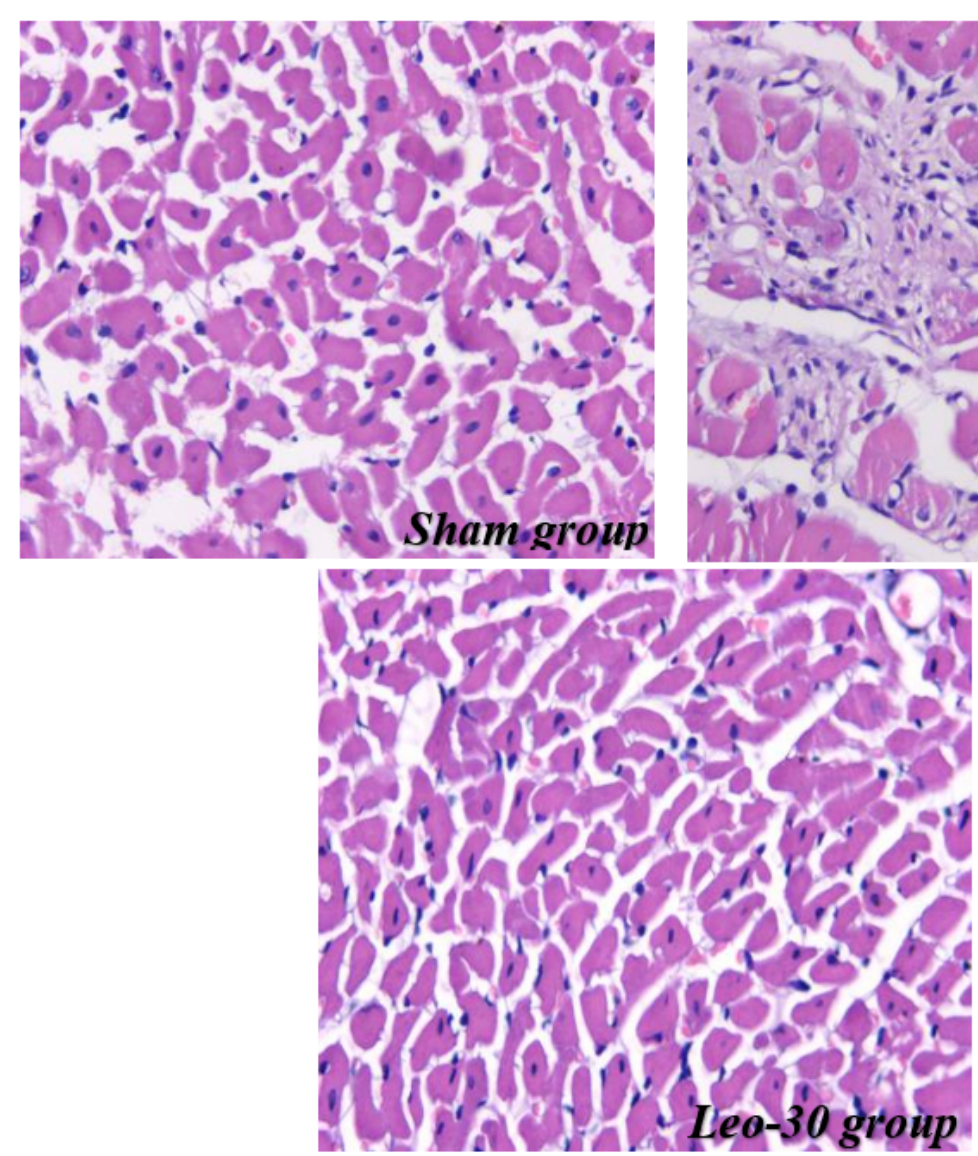
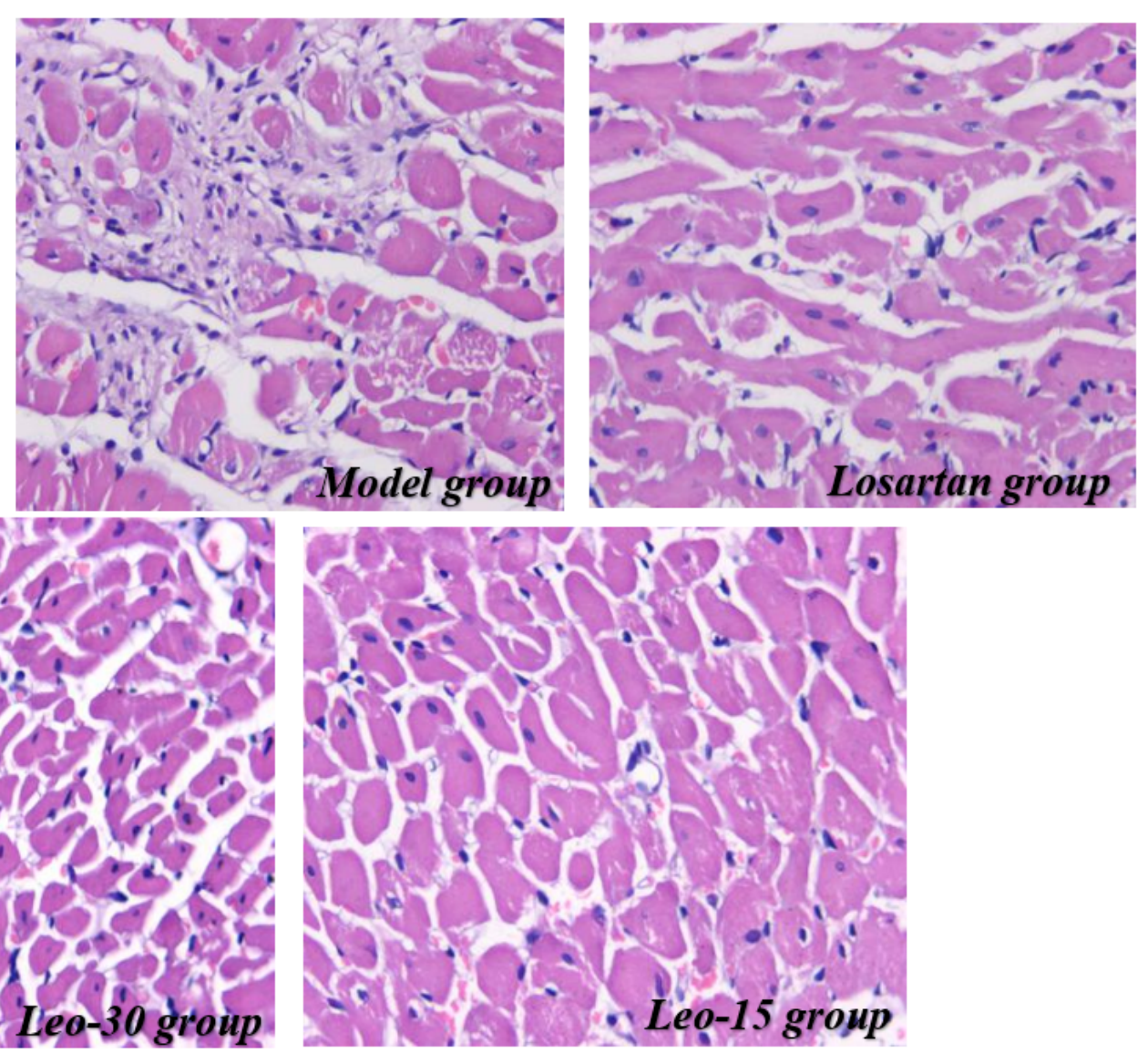

Figure 2

Effects of leonurine on the tissue in left ventricular myocardium 


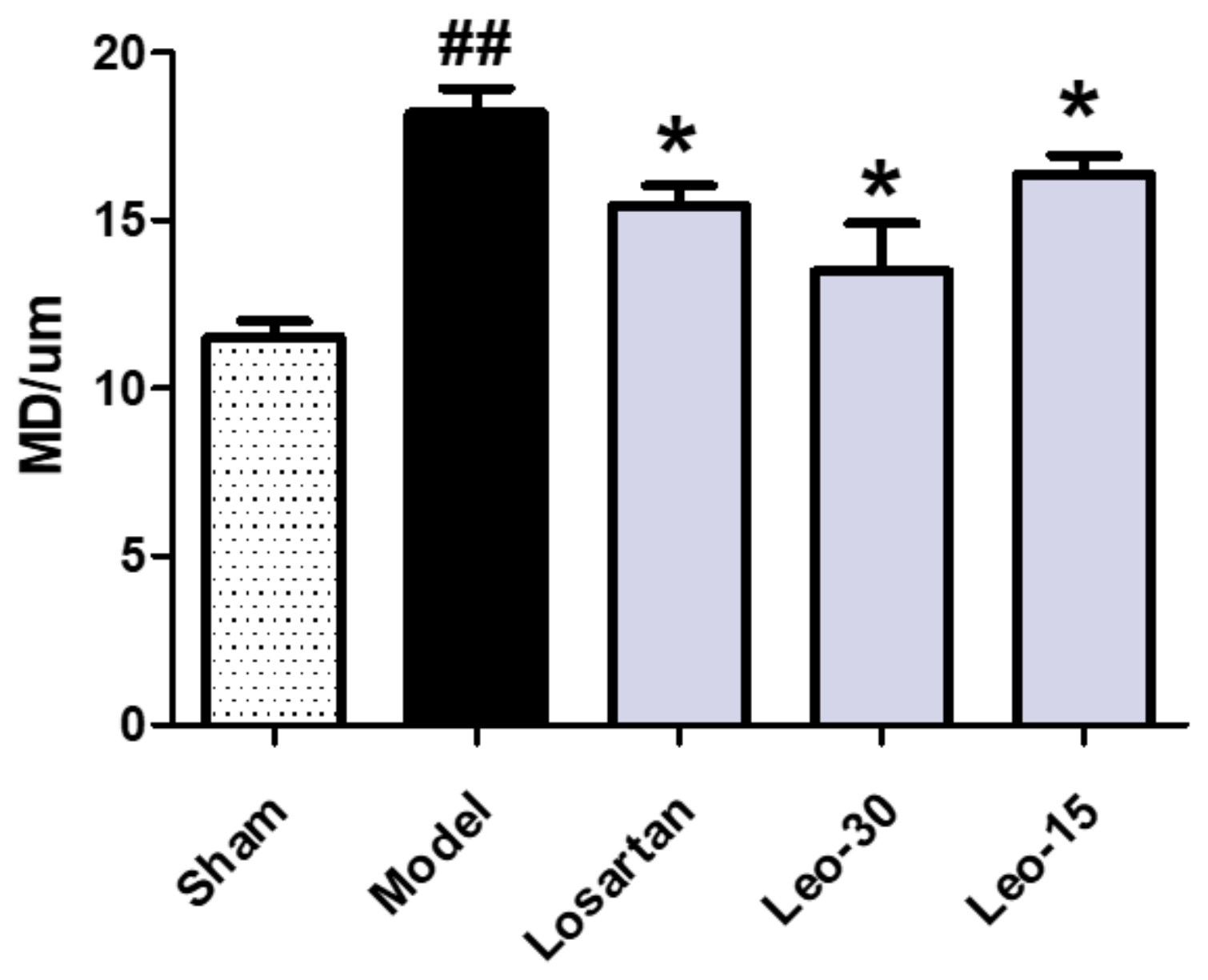

Figure 3

Effects of leonurine on cardiac myocyte diameter MD, the size of myocardial cell diameter.Data are means $\pm s . d, n=8$ rats. \#\#p 0.01 vs Sham group;*p $₫ 0.05$ vs Model group.
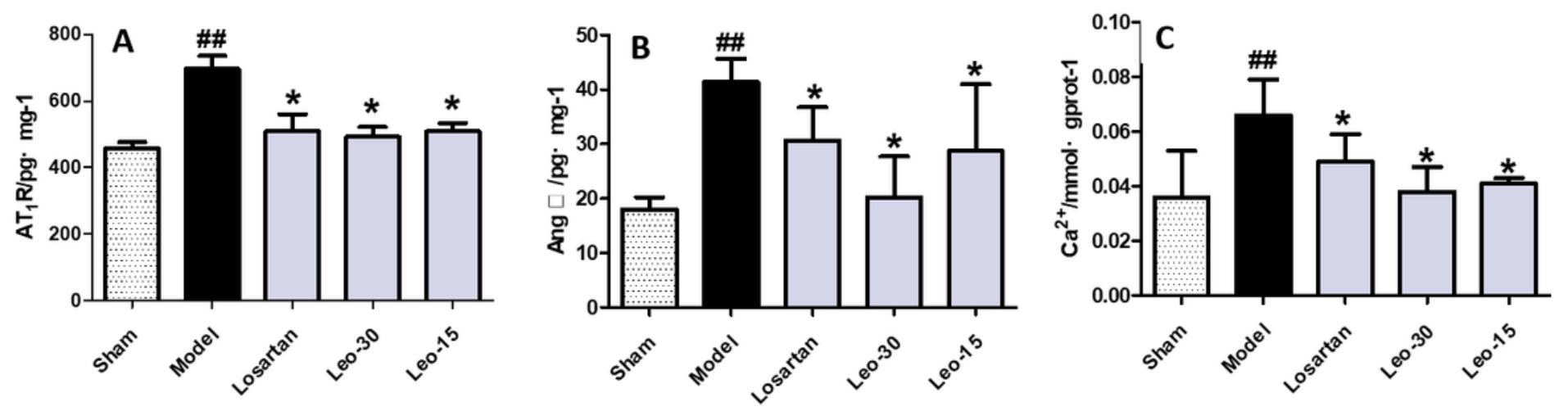

Figure 4

Effects of Leonurine on the contents of Angll, AT1R and Ca2+ in myocardial tissue Ang II, angiotensin II,

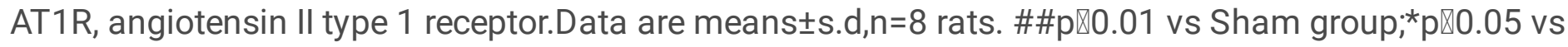
Model group. 
A

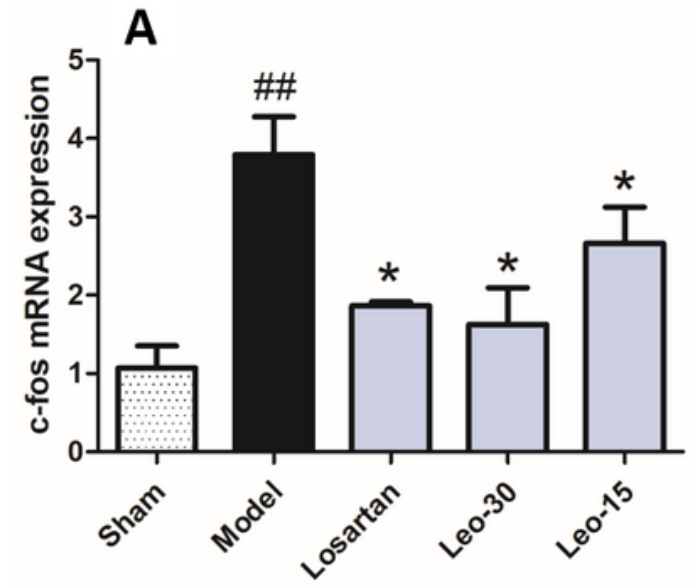

C
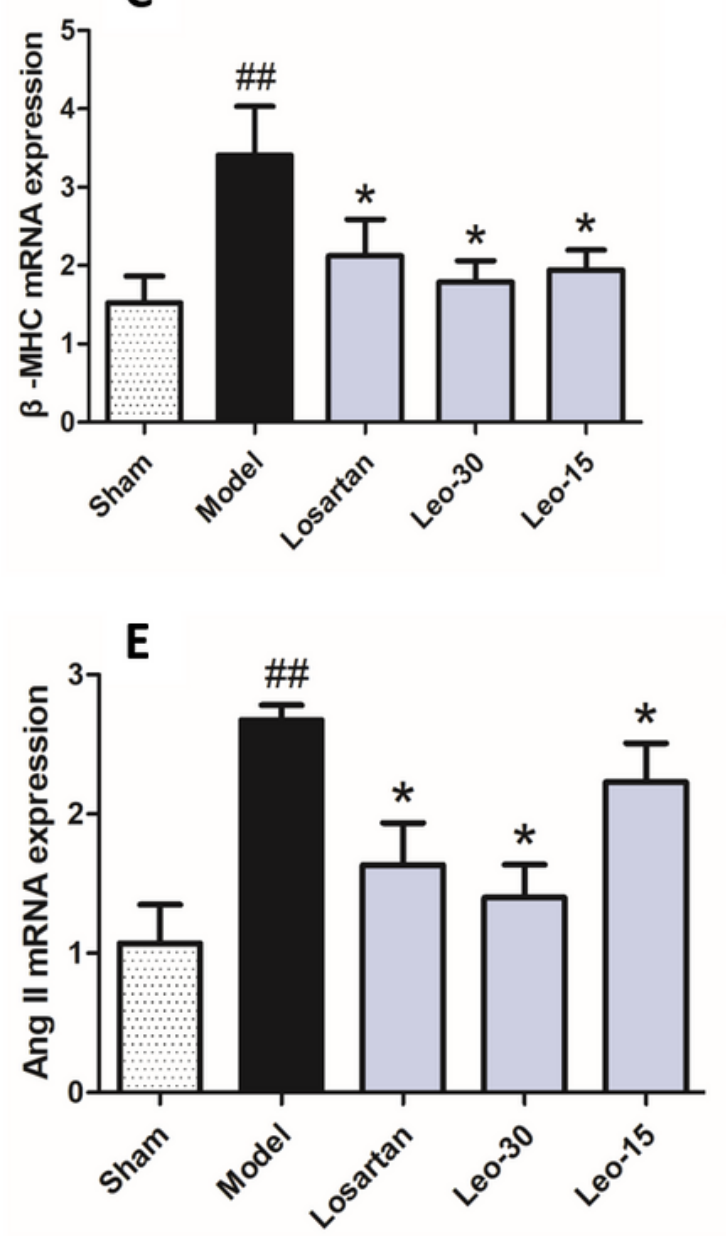

B
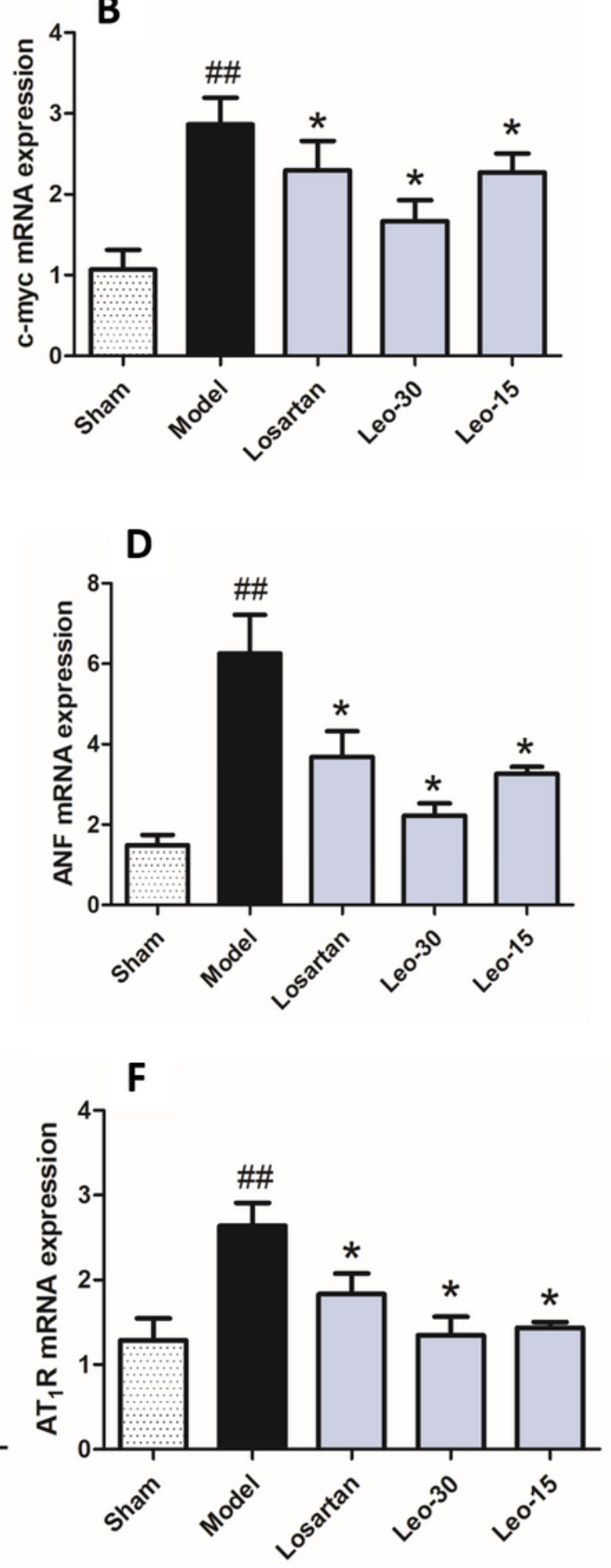

Figure 5

Effects of leonurine on the expression of $\beta$-MHC, ANF, c-fos, c-myc, Angll and AT1R mRNA in left ventricular myocardium ANF, atrial natriuretic factor, $\beta-M H C, \beta$-myosin heavy chain.Data are

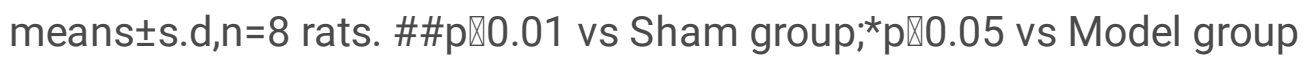


A
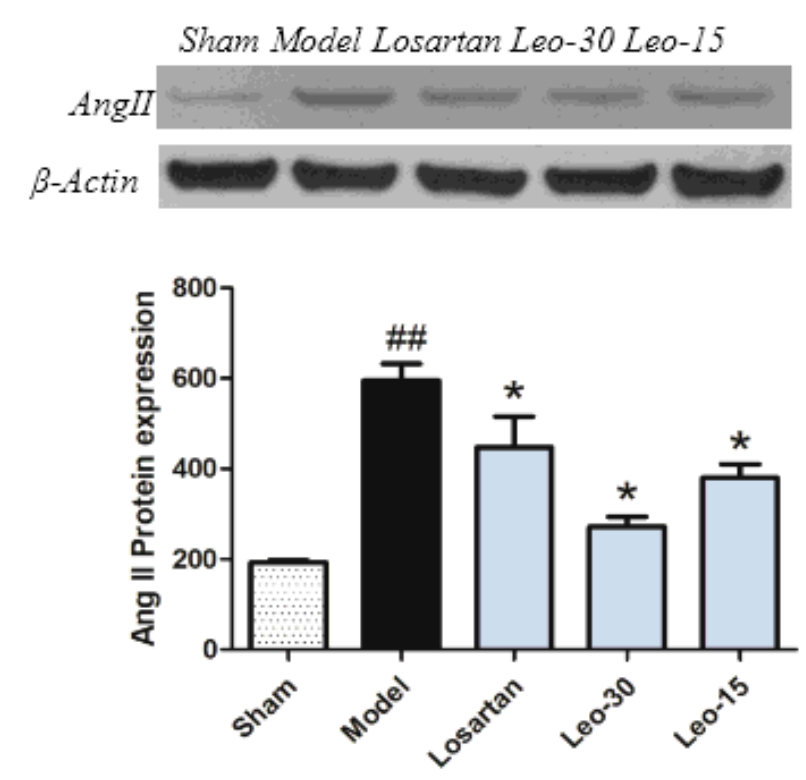

C
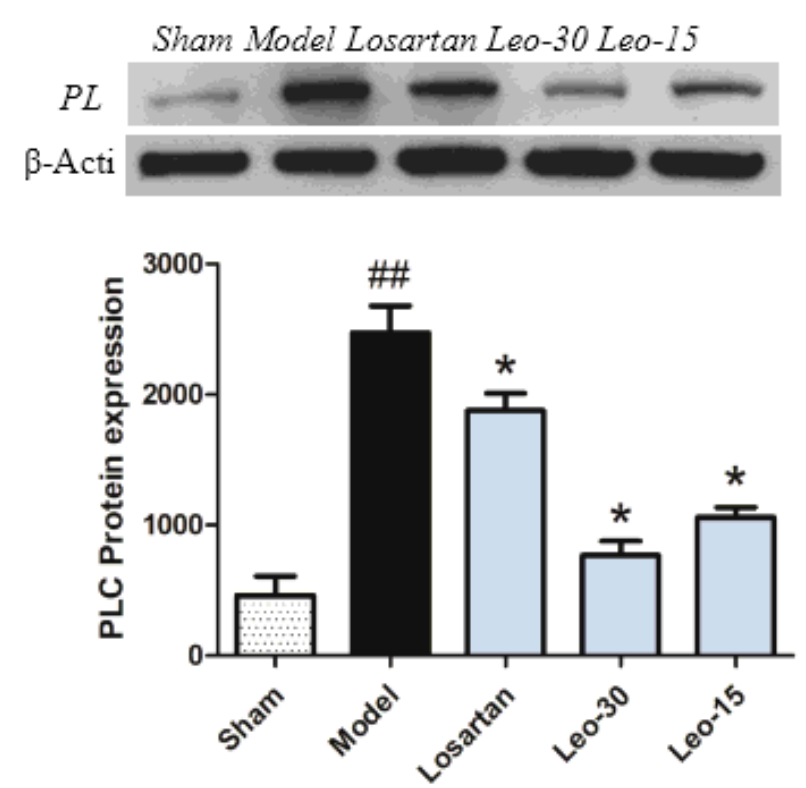

B

Sham Model Losartan Leo-30 Leo-15
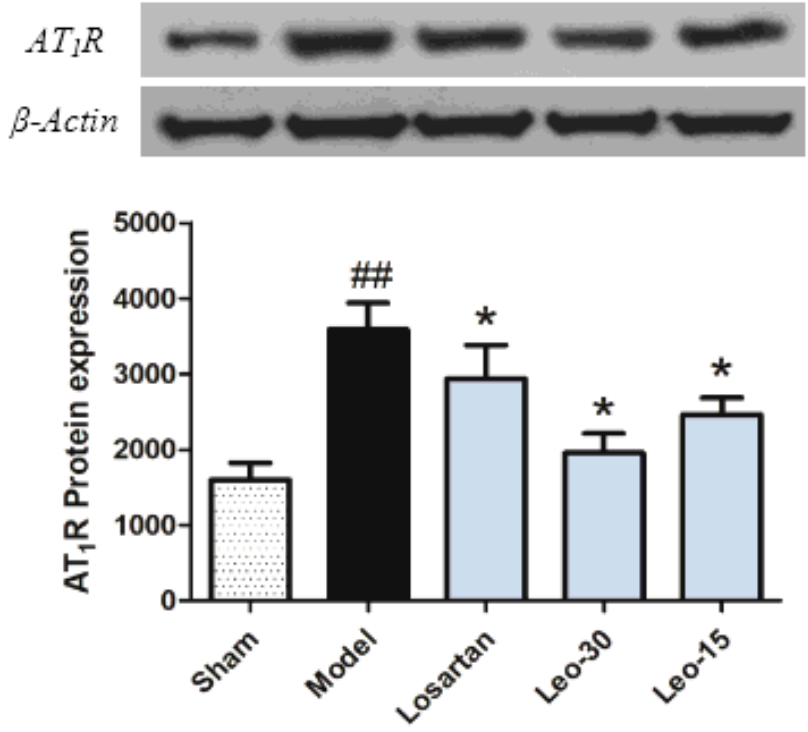

D
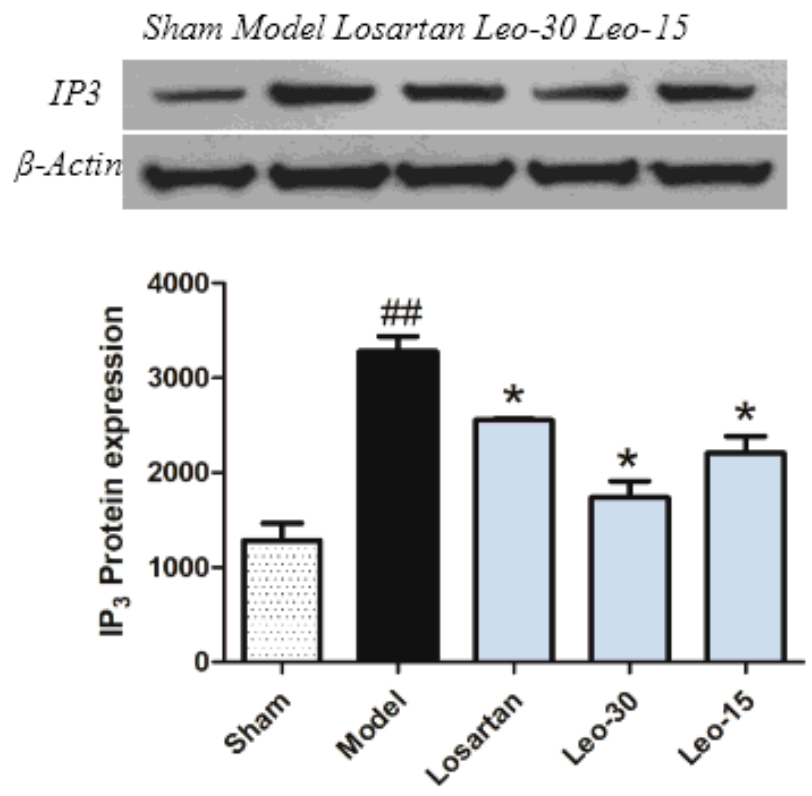

Figure 6

Effects of leonurine on the expression of Angll, AT1R, PLC and IP3 protein in left ventricular myocardium

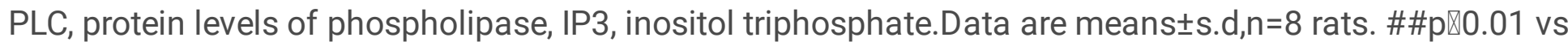
Sham group;*p $₫ 0.05$ vs Model group. 